\title{
Uma Aplicação Web para Representação de um Guia de Participação Pública
}

\author{
Dênis S. Rosa ${ }^{1}$, Bárbara P. Caetano ${ }^{2}$, Melise M. V. Paula ${ }^{1}$, Jano M. de Souza² \\ ${ }^{1}$ Universidade Federal de Itajubá (UNIFEI) \\ Caixa Postal 50 - 37500-903 - Itajubá- MG - Brazil \\ ${ }^{2}$ Universidade Federal do Rio de Janeiro (UFRJ) \\ Caixa Postal 68.010 - 96201-900 - Rio de Janeiro - RJ - Brazil \\ \{denis.souza.rosa, melise\}@unifei.edu.br, \\ \{bpimentacaetano, jano\} @cos.ufrj.br
}

\begin{abstract}
In recent years, the advancement and popularization of technology have expanded opportunities for citizen participation in discussions related to politics and society. However, citizens still have little knowledge about their rights and duties in this scenario. Although it is possible to find some initiatives that try to solve the challenge found, some progress is still needed. This work is part of a project that aims to propose a guide that facilitates the sharing of knowledge about social participation. The purpose of this article is to propose a visual representation for the guide that facilitates the interpretation of information and user interaction. For validation, interviews were conducted with potential users, in which it was possible to identify the necessary improvements to facilitate the understanding of the information.
\end{abstract}

Resumo. Nos últimos anos, o avanço e a popularização da tecnologia ampliaram as oportunidades de participação do cidadão nas discussões relacionadas à política e a sociedade. No entanto, os cidadãos ainda têm pouco conhecimento sobre seus direitos e deveres neste cenário. Embora seja possivel encontrar algumas iniciativas que tentem resolver o desafio encontrado, ainda são necessários alguns avanços. Este trabalho faz parte de um projeto que tem como objetivo propor um guia que facilite o compartilhamento do conhecimento sobre a participação social. O objetivo deste artigo é propor uma representação visual para o guia que facilite a interpretação das informações e a interação do usuário. Para validação foram realizadas entrevistas com possíveis usuários, em que foi possível identificar as melhorias necessárias para facilitar o entendimento das informações.

\section{Introdução}

A participação do cidadão em discussões relacionadas à política e a sociedade está aumentando nos últimos anos. Há diferentes situações nas quais a população participou de forma definitiva em processos importantes. No trabalho de Santos et al. (2019), por exemplo, os autores descrevem como a população teve papel fundamental na revisão do plano diretor de Porto Velho - RO. Em Macedo et al. (2019), os autores avaliaram o desempenho do comitê comunitário no plano diretor de Itaguaí - RJ. Já em Leite et al. (2018), o autor mostra como a população contribuiu para a revisão do plano diretor da cidade de Pirapora - MG. 
Contudo, para que a participação seja efetiva, ainda existem vários desafios que devem ser considerados. Um dos desafios é a falta de conhecimento dos cidadãos sobre seus direitos e deveres. Na tentativa de contribuir para solucionar esse desafio, está sendo elaborado um guia para facilitar a formalização do conhecimento sobre a participação social e o entendimento de gestores e cidadãos sobre o tema.

Para estruturar a informação coletada de forma organizada, foi investigada uma estratégia para modelagem dos dados. Nessa análise, constatou-se que a relação entre os elementos desse conjunto de dados assemelha-se a um grafo. Dessa forma, optou-se por utilizar um banco de dados orientado a grafo sendo escolhido o Neo4j. Esse banco de dados é uma abordagem NoSQL que define uma estrutura de baixa rigidez que pode ser constantemente remanejada na fase de desenvolvimento de uma aplicação (Santos and Silva, 2013).

Além disso, outra vantagem em usar essa abordagem é a performance para realizar consultas a partir da representação dos dados como um conjunto de vértices e arestas que facilitam o acesso às informações (Silva, 2016). Esses são requisitos importantes para o domínio desse trabalho. Outro aspecto importante é a visualização desses dados, sendo necessária a utilização de alguma estratégia que, combinada ao mecanismo de persistência, disponibilize essa informação de maneira que diferentes perfis de usuários possam explorar o conhecimento formalizado na estrutura.

Desta forma, o foco principal deste trabalho é propor uma representação visual do guia que facilite a interpretação e exploração da informação por meio de um ambiente colaborativo, no qual o usuário poderá interagir com o sistema e propor mudanças com o intuito de evoluir essa representação. Para isso, será utilizada uma abordagem para análise visual de dados de forma que sejam determinadas técnicas de visualização adequadas ao domínio. Vale destacar que esse trabalho está sendo desenvolvido no contexto de um projeto de pesquisa cujo objetivo é promover a participação social por meio de diferentes iniciativas e uma delas é a elaboração do guia. Contudo, o guia propriamente dito não é objeto desse artigo, mas sim a representação visual dos dados que já foram coletados.

\section{Trabalhos Relacionados}

Nos últimos anos muitos trabalhos vêm sendo desenvolvidos com o objetivo de analisar os aspectos motivacionais e solucionar a falta de engajamento dos cidadãos. Berntzen e Johannessen (2016), por exemplo, apresentam uma visão geral das tecnologias utilizadas em ferramentas de participação que engajem os cidadãos. Já o trabalho realizado por Hassan et al. (2018), por exemplo, apresenta um estudo de caso de um projeto para criar uma ferramenta de e-participação gamificada.

Como forma de estruturar as tecnologias e ferramentas utilizadas para o engajamento, no trabalho de Mota et al. (2020) foi apresentada uma representação visual para classificação de ferramentas de participação eletrônica. De acordo com os autores, o grande desafio foi a escolha da técnica de visualização e a classificação, sendo elaborada por meio da análise das ferramentas encontradas e da revisão da literatura.

No que diz respeito ao uso da interface para exposição do conhecimento, a utilização de grafos para uma representação visual pode ser encontrada em outros trabalhos, como no de Ramos et al. (2016), no qual é descrito um modelo para a trilha de aprendizagem dos alunos, sendo exibido para os docentes e tutores como uma ferramenta capaz de auxiliar no desenvolvimento dos alunos em classe. Já no trabalho de Carvalho 
et al. (2014), os autores apresentam uma proposta para classificar as opiniões de clientes em relação a um conjunto de restaurantes. Os autores afirmam que as técnicas de visualização em grafo contribuíram para a tomada de decisão tanto para os gestores dos restaurantes, quanto para futuros clientes. Esses trabalhos possuem uma característica comum pois tiveram como desafio a representação da informação. Demonstrando assim como a visualização da informação pode auxiliar no entendimento e na tomada de decisão.

\section{Guia de Participação}

A estrutura do guia considera alguns elementos que têm como finalidade caracterizar a participação pública. O grafo da Figura 1, denominado matriz, ilustra as relações entre esses elementos.

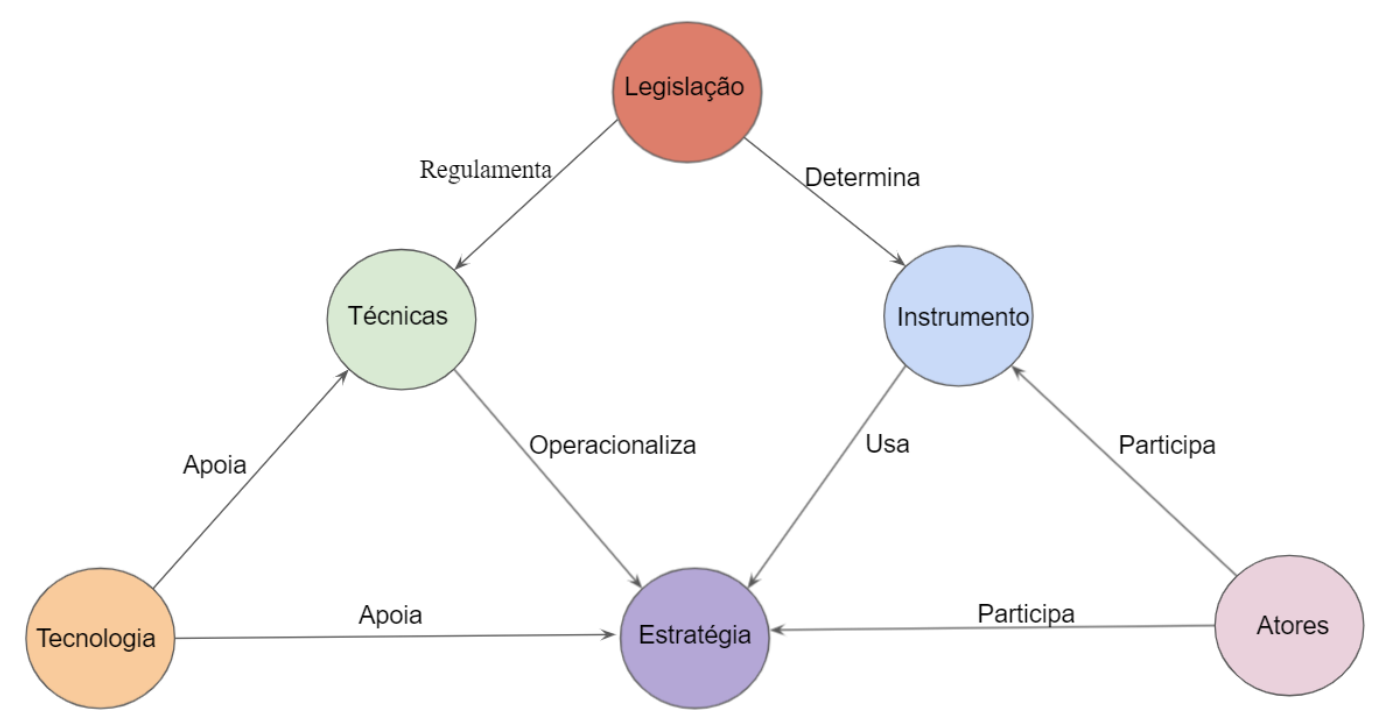

Figura 1. Matriz. Fonte: Autoria Própria

A matriz contém uma estrutura de vértices e arestas que representam os dados identificados no guia. Os vértices foram divididos em grupos. Cada grupo representa uma unidade de informação que tem um papel específico no contexto. A forma como esses grupos se relaciona é definida pelas arestas. Os grupos definidos foram: Legislação: é composto pelas leis e pode se relacionar com as técnicas e os instrumentos. Uma lei pode determinar os instrumentos de participação e pode ser associada a uma técnica; Instrumento: é composto pelos diversos tipos de instrumentos de participação que são usados para cumprir uma determinada lei. A participação em cada instrumento pode ser operacionalizada utilizando técnicas diferentes. Como exemplo, considere o Plano Diretor Municipal Participativo (PDM). O PDM é um instrumento adotado pela gestão pública para definir o direcionamento da política urbana e desenvolvimento estrutural Leite el al. (2018). A Lei Federal $n^{\circ} 10.257 / 01$, regulamentada por meio do Estatuto da Cidade, determina que o PDM é obrigatório para as cidades com mais de vinte mil habitantes. Segundo Ferreira et al. (2019) a elaboração do PDM deve ser realizada por meio da efetiva participação social em todas as fases de seu desenvolvimento; Técnica: representa as técnicas que podem ser usadas para que a participação seja efetiva nos instrumentos definidos pelas leis, por exemplo, nos Planos Diretores Participativos, é necessário que sejam realizadas audiências públicas. Outros exemplos de técnicas são os 
plebiscitos e o voto; Ator: os atores representam os agentes envolvidos nesse processo de participação. Como exemplos é possível citar o poder público e a sociedade que são, naturalmente, atores em todos os processos de participação modelados. Mas em alguns contextos a sociedade pode ser segmentada em diversos grupos como, ONGs, associações comerciais, população de zona rural e urbana, empresas dentre outros. O mesmo acontece com o poder público que pode ser representado, por exemplo, pelo gestor de uma cidade ou pelo ministério público; Tecnologia: esse grupo representa qualquer tecnologia que pode ser usada para a execução de uma determinada técnica. Pode-se citar ferramentas de participação eletrônica, o voto eletrônico, ou ainda, no cenário de isolamento social, ferramentas para reuniões assíncronas e síncronas para as audiências públicas virtuais que têm sido utilizadas como alternativas para a operacionalização de alguns instrumentos; Estratégia: como o próprio nome já diz, é a estratégia utilizada durante a execução do instrumento para mobilizar o engajamento dos atores, apoiar as tecnologias utilizadas e operacionalizar as técnicas definidas.

\section{Descrição da proposta}

Para a representação do guia de participação pública está sendo proposta uma aplicação web que permita ao usuário interagir com a interface elaborada para a visualização da matriz, que é a representação dos agrupamentos identificados. Além de visualizar essa estrutura, deverá ser possível percorrer a matriz ao selecionar o agrupamento desejado, exibindo todos os vértices associados.

Além da representação visual e a navegabilidade, a aplicação está sendo elaborada como um ambiente colaborativo no qual todos os usuários poderão participar da evolução do Guia. O ambiente colaborativo surge como uma alternativa para motivar os potenciais usuários, uma vez que potencializa a percepção de co-autoria, podendo motivar os cidadãos a participarem desse processo de construção desse conhecimento, além de poder aumentar o engajamento dessas pessoas na elaboração de políticas públicas (Vaz, 2011).

O usuário poderá interagir com essa interface podendo percorrer toda a estrutura da matriz, sugerir alterações e comentar sobre diferentes tipos de agrupamentos apresentados. Contudo, as alterações na estrutura do guia serão controladas, as sugestões deverão ser direcionadas a um moderador do sistema, que terá o papel fundamental em analisar as sugestões, podendo aceitar ou não.

Portanto, é necessário e essencial que aspectos de usabilidade sejam cuidadosamente considerados no projeto da interface entre os usuários e os elementos visuais que representam os dados. Além das questões visuais, deve-se considerar os aspectos relacionados à colaboração. Dessa maneira, foram identificados os seguintes requisitos (ou desafios): Evoluir o guia: A evolução do guia com a alteração da matriz. Nesse caso, o usuário poderá sugerir a criação de um novo agrupamento ou um novo relacionamento entre os agrupamentos apresentados. Exemplo: considere o agrupamento Estratégia, no qual deve ser definida a estratégia utilizada para que a participação seja efetiva no instrumento executado. Esse agrupamento não havia sido identificado na primeira versão do grafo tendo sido reconhecido ao discutir o contexto com alguns especialistas em gestão pública. Considerando os aspectos relacionados à interface, é necessário que o usuário possa interagir com os vértices. Ao clicar em um vértice, deverá ser apresentado um detalhamento do grupo contendo sua descrição. Além disso, o usuário poderá visualizar os agrupamentos que foram sugeridos por outros usuários, mas que 
ainda estivessem em análise do moderador. Outros usuários poderão comentar as sugestões propostas, contribuindo também para a evolução da ideia; Instanciar o guia: O usuário poderá instanciar o guia a partir da matriz adicionado novos elementos em cada um dos grupos, por exemplo: cadastrar um plano diretor de uma cidade, adicionando as leis os instrumentos e todos os recursos necessários para a criação desse plano. $\mathrm{Na}$ interface, deverão ser considerados recursos que permitam que o usuário identifique de forma intuitiva como instanciar elementos para cada grupo e criar relacionamentos entre eles. Além disso, deverão ser considerados aspectos que facilitem a identificação do agrupamento de cada instância como o uso de cores distintas; Visualizar o guia: Será possível ver a matriz do guia que contém todos os grupos identificados que são diferenciados por cor; Consultar agrupamento: ao selecionar um grupo na matriz, todos os atributos relacionados a esse grupo serão apresentados juntamente com suas arestas; Percorrer grafo: Quando o usuário selecionar um dos grupos, serão exibidos todos os vértices do grupo, ao escolher um desses vértices, serão exibidos todos os elementos que se relacionam com o vértice selecionado, como exemplo, considere o vértice (grupo) Legislação, ao clicar nesse grupo, deverão ser exibidas todas as instâncias da legislação identificadas (leis, decretos, dentro outros que preconizam a participação), ao selecionar um dos "filhos" do grupo Legislação, Estatuto das Cidades, por exemplo, serão exibidos todos os filhos de outros grupos que se relacionam com esse vértice; Comentário: Os usuários poderão comentar ou fazer sugestões em relação à evolução do Guia de forma a aumentar as possibilidades de colaboração.

Em relação às classes de usuários, foram identificados inicialmente três perfis como possíveis usuários. Cidadão, que é um usuário comum e não possui conhecimento sobre participação ou como pode participar das discussões relacionadas às políticas públicas; o gestor, que é responsável por proporcionar essas oportunidades de participação e equipe técnica, que representa o grupo de atores que são responsáveis pela elaboração desses instrumentos e, consequentemente, execução das técnicas definidas. A equipe técnica e a gestão pública são papéis que podem ser executados pelas mesmas pessoas. Outra alternativa comum nesse contexto é o gestor público contratar um serviço terceirizado para a elaboração desses instrumentos.

\subsection{Prototipação}

Nesta etapa do trabalho, foi elaborado um protótipo para facilitar a discussão e análise da primeira proposta para a representação do guia. Neste protótipo, foi representada a forma como os agrupamentos deverão compor a matriz, além de simular alguns comportamentos da aplicação. Os seis tipos de agrupamentos foram diferenciados pelas cores e os relacionamentos entre os grupos são representados pelas arestas. Além disso foi previsto um menu lateral para facilitar a navegação e auxiliar na identificação das cores dos grupos. Quando é selecionado algum agrupamento na matriz do guia ou no menu, são exibidos todos os vértices (filhos) referentes ao grupo escolhido.

No protótipo, foram implementadas algumas funcionalidades. O guia é apresentado ao lado da legenda permitindo visualizar todos os grupos. A figura 2 ilustra as informações apresentadas quando o grupo Técnica é selecionado, ou seja, até o momento, três técnicas foram identificadas para operacionalização das estratégias definidas para a mobilização e participação social: discussões e debates, oficina estratégica e audiência pública. 


\section{Legenda}

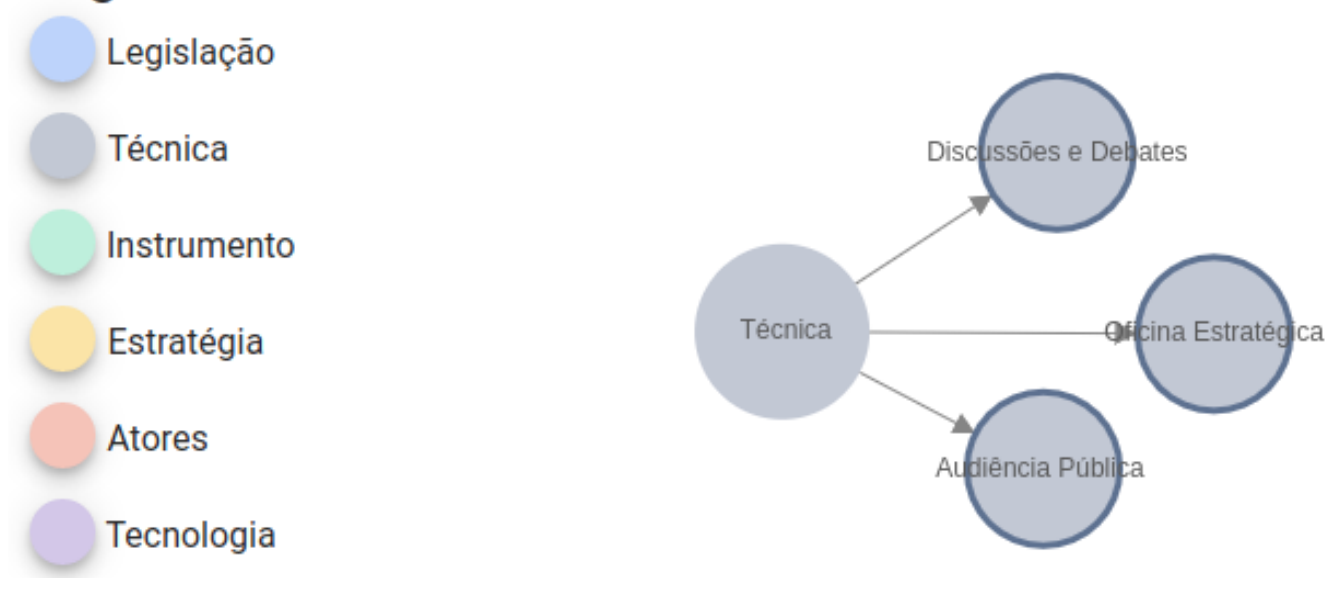

Figura 2. Agrupamento das Técnicas. Fonte: Autoria Própria

\section{Avaliação do protótipo}

Para validar o protótipo proposto, foi realizado uma avaliação na qual foi utilizado um questionário para conduzir uma entrevista. A escolha da amostra não foi aleatória, participaram três pessoas que atualmente trabalham em projetos que envolvem a elaboração de políticas públicas, que exigem a participação social. Para direcionar a entrevista, foram definidas perguntas. Algumas dessas perguntas questionam pontos específicos da interface que demandam uma investigação pois há dúvida em relação a sua aceitação. As perguntas definidas foram: P1: Como você avalia seu conhecimento em participação pública? P2: Você concorda que as interações com o guia foram de fácil entendimento? P3: Você concorda que a borda mais escura de cada elemento no guia auxilia percorrer toda a estrutura? P4: Você concorda que as cores usadas para diferenciar cada agrupamento ajudaram no entendimento de como estão organizados? P5: Você concorda que o menu lateral "Legenda" auxiliou na navegação? P6: Você concorda que os recursos visuais da ferramenta proporcionam uma experiência agradável? P7: Você usaria novamente o Guia SoPa, ou indicaria para um amigo? P8: No seu entendimento, ficou claro o que são os agrupamentos e como eles se relacionam? P9: Na sua visão, a forma com que a informação está apresentada faz sentido pra você? Se não, o que poderia ser diferente? P10: Você considera que essas informações são úteis, de alguma forma, pra você? Como? P11: Há alguma informação sobre esse contexto, que não está presente no guia, que você acha interessante adicionar? P12: Você saberia dizer o que a estratégia representa no guia?

Todos os participantes afirmaram que a participação pública é um assunto pelo qual se interessam. Quando os participantes interagiram com o guia nenhum afirmou ser de fácil entendimento, o que pode indicar que a forma de apresentação da informação precisa de melhorias. Uma das pessoas entrevistadas afirmou ter tido dificuldade para interagir com a ferramenta. Esse participante não reconheceu a ajuda das bordas destacadas para navegabilidade. As outras duas pessoas afirmaram que as bordas mais escuras ajudaram a percorrer a estrutura do grafo. Além disso, essas mesmas pessoas reconheceram a influência das cores no reconhecimento dos grupos. Em relação à legenda, todos afirmaram que o menu lateral foi reconhecido como um facilitador. 
Apesar de um participante discordar que os recursos visuais da ferramenta proporcionam uma experiência agradável, dois participantes concordaram que a interface é visualmente agradável.

Ao serem questionados se usariam novamente o guia ou se indicariam para algum amigo, apenas uma pessoa respondeu com certeza que indicaria ou usaria novamente a ferramenta.

Quando os participantes foram questionados sobre os agrupamentos e seus relacionamentos apenas uma pessoa não compreendeu, alegando ter muita informação exibida na interface, o que confundia o entendimento. Todos concordaram que a informação exibida é útil, pois pode auxiliar na explicação do conteúdo para pessoas que ainda não possuem esse conhecimento específico. Além disso um dos participantes afirmou que o guia pode ajudar líderes a ter uma visão mais ampla de todo o contexto, de um plano diretor, por exemplo.

Sobre adicionar mais informação no guia apresentado todos os participantes afirmaram que seria interessante adicionar descrição sobre os vértices, principalmente sobre os instrumentos apresentados.

Analisando as respostas foi possível identificar quais melhorias são necessárias para facilitar o entendimento da informação apresentada. A legenda com as cores facilitou o entendimento dos agrupamentos, porém foi possível observar que a navegabilidade no guia precisa de melhorias, pois os usuários afirmaram ficar confusos com a quantidade de informação apresentada quando muitos vértices foram clicados. Nesse caso, foi sugerido que fossem mostrados menos níveis do grafo ou que o caminho percorrido fosse destacado ao navegar pelo guia. Entretanto, se por um lado mostrar menos níveis diminui a quantidade de informações, destacar o caminho percorrido aumenta a quantidade de informações apresentadas na interface.

As descrições dos vértices serão adicionadas conforme sugerido e, além disso, outras soluções serão avaliadas e implementadas para melhoria da representação como, por exemplo, a utilização de breadcrumbs para representar a navegação estrutural do guia.

\section{Considerações finais}

O trabalho descrito neste artigo está em andamento e visa construir um ambiente colaborativo que disponibilize uma representação visual para o Guia de Participação e que facilite o compartilhamento do conhecimento representado. Para isso, como estratégia de implementação, foi utilizando um modelo de persistência e técnicas de visualização baseadas em grafo.

No artigo, foi apresentada a representação visual proposta, definida como uma estrutura matriz, que representa os diversos grupos de informação coletados e os vértices, que podem ser considerados como pertencentes aos agrupamentos identificados. Além disso, foi descrito também aspectos sobre o comportamento dessa matriz quando o usuário realiza suas interações. Como forma de avaliação foi realizado uma entrevista em que foi possível identificar necessidades de melhorias. Como evolução do trabalho, a aplicação será finalizada considerando os resultados da avaliação. Além disso serão realizadas novas avaliações considerando os diferentes perfis de usuários identificados. 


\section{Referências}

Berntzen, L., \& Johannessen, M. R. (2016). The role of citizen participation in municipal smart city projects: Lessons learned from Norway. In Smarter as the new urban agenda (pp. 299-314). Springer, Cham.

Carvalho, E. S., Chaves, M. S. and Sacramento, N. (2014). "Visualização de informação de opiniões online sobre restaurantes: uso de técnicas orientadas à visualização de grafos". Revista eletrônica de sistema de informação. 13(1).

Ferreira, G., JESUS, I. S. and Mariano, M. V. (2009). "Plano diretor e a participação pública no planejamento urbano", Âmbito jurídico. Rio Grande. XII, (65).

Hassan, L., Morschheuser, B., Alexan, N., and Hamari, J. (2018). First-hand experience of why gamification projects fail and what to do about it. In Proceedings of the 2nd International GamiFIN Conference (GamiFIN2018), Pori, Finland, May 21-23, 2018. Gamification Group.

Leite, Dias, H. V. (2018). “A importância da revisão do plano diretor municipal: Um estudo na cidade de Pirapora - MG”.

Macedo, L. F. d. A. (2019). “Atuação dos conselhos comunitários no acompanhamento e participação cidadã no plano diretor de desenvolvimento sustentável do município de Itaguaí - RJ".

Mota, F. B. d. S., Paula, J. H. R., Caetano, B. P., Paula, M. M. V. and Souza, J. M. (2020). "TAPE: Taxonomia de Aplicações em Participação Eletrônica". Revista Tecnologia e Sociedade.16(42): 207-226.

Ramos, D. B. (2016). Uma ferramenta baseada em grafo para identificação e visualização de trilhas de aprendizagem.

Santos, E. U., and SILVA, M. (2013). Abordagem ao Banco de Dados Orientado a Grafos Neo4j em um Nível Empresarial.

Santos Junio, S., Duarte, J. L., \& de Souza, L. D. P. (2019). Participação popular no Plano Diretor de Porto Velho, Rondônia-Brasil. Revista Políticas Públicas \& Cidades-23591552, 8(4).

Silva, G. H. M. A. D. (2016). Um modelo de visualização de dados utilizando banco de dados orientado a grafo suportado por big data.

Vaz, A. C. N. (2011). Participação política, efeitos e resultados em políticas públicas: notas crítico-analíticas. Opinião Pública. 17(1), 163-205. 